\title{
La Société suisse de médecine intensive (SGI-SSMI)
}

Prof. Lucas Liaudet, secrétaire de la SGI-SSMI

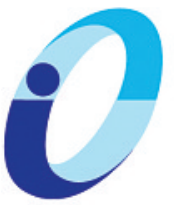

SCHWEIZERISCHE GESELLSCHAFT FÜR INTENSIVMEDIZIN SOCIÉTÉ SUISSE DE MÉDECINE INTENSIVE SOCIETÀ SVIZZERA DI MEDICINA INTENSIVA SGI-SSMI-SSMI

\section{Introduction}

La Société suisse de médecine intensive a été fondée le 4 février 1972 à Bâle. De 28 membres fondateurs à ses débuts, elle compte aujourd'hui 453 membres et 88 unités de soins intensifs certifiées. Notre société est affiliée à la FMH ainsi qu'à la fmCh, l'Association suisse des médecins avec activité chirurgicale et invasive. Ses objectifs prioritaires sont la garantie d'une formation postgraduée et continue de haute qualité, la promotion de la recherche et la défense des intérêts professionnels de ses membres.

\section{Organisation et missions de la SGI-SSMI}

Diverses commissions gèrent les tâches principales de la société. La commission de reconnaissance des unités de soins intensifs (USI) a établi des directives pour la planification de nouvelles USI et la restructuration d'USI existantes, qui correspondent aux exigences minimales en termes d'architecture, d'organisation et de qualité d'une USI. La commission des données a élaboré le concept des «données minimales», qui donnent des informations essentielles sur la qualité des structures, des processus et des résultats d'une USI et qui sont nécessaires pour la certification de l'USI, sa reconnaissance pour la formation, ainsi que pour la catégorisation Diagnosis Related Groups. La commission de la formation postgraduée et continue procède à la révision régulière du programme de formation en médecine intensive et réévalue régulièrement les établissements de formation. La commission du congrès est responsable de la promotion de la recherche en médecine intensive en Suisse. Elle se compose de huit membres dont trois secrétaires scientifiques qui planifient les assemblées annuelles de la Société et en organisent les séances. La commission d'examen est responsa- ble de l'organisation annuelle des examens de spécialiste en médecine intensive. Elle procède à l'élaboration des questions d'examen et établit une liste d'experts qualifiés pour évaluer les candidats. La commission paritaire de la formation postdiplôme d'infirmier (-ère) en soins intensifs, formée de délégués médicaux de la SGI-SMI et de délégués de l'association suisse des infirmiers (-ères) avec diplôme de formation en soins intensifs, est l'organe compétent en matière de réglementation et de surveillance de la formation des infirmiers (-ères) en soins intensifs.

Notre société est également dotée d'une commission tarifaire et d'une commission sur le don d'organes et la transplantation, ainsi que de groupes d'intérêts ayant pour but de promouvoir la prise en charge, la formation, la recherche et la qualité dans leur domaine d'intérêt. La communauté d'intérêts suisse de soins intensifs de l'association professionnelle des infirmiers (-ères) représente les intérêts de ses membres dans le domaine des soins intensifs. Le groupe d'intérêt pour le sepsis, composé de membres de différentes sociétés a pour but la création de réseaux entre les cliniques, les instituts de recherche et l'industrie, le développement de directives pour le diagnostic et le traitement du sepsis, et la mise en œuvre de la formation dans ce domaine.

\section{Assemblée annuelle}

La Société organise de nombreux symposiums, ateliers de formation et un congrès annuel, conjointement avec une autre société de discipline médicale. Le prochain congrès se tiendra à St-Gall du 24 au 26 septembre 2009, avec les Sociétés suisses de neurochirurgie et de neuroradiologie. Pour de plus amples informations, nous vous invitons à consulter nos sites internet:

- www.sgi-ssmi.ch;

- Assemblée annuelle 2009 à St-Gall: http:// kongress2.imk.ch/sgi2009/WebHome. 